\title{
Un nuevo género de coleóptero acuático de Venezuela (Hydradephaga: Noteridae)
}

\author{
Mauricio García ${ }^{1 *}$ \& Jesús Camacho² \\ 1. Centro de Investigaciones Biológicas, Facultad de Humanidades y Educación, Universidad del Zulia, Apartado 526, Maracaibo A-4001, Estado \\ Zulia, Venezuela; meruidae2014@gmail.com \\ 2. Museo de Artrópodos, Departamento Fitosanitario, Facultad de Agronomía, Universidad del Zulia, Apartado 15205, Maracaibo 4005, Estado \\ Zulia, Venezuela; jcamacho@fa.luz.edu.ve \\ * Correspondencia
}

Recibido 24-VIII-2017 • Corregido 19-X-2017 • Aceptado 23-X-2017

\begin{abstract}
A new genus of aquatic coleopteran from Venezuela (Hydradephaga: Noteridae). Suphisellus is a Nearctic genus of aquatic coleopterans that has been misidentified in the tropics. We analyzed "Suphisellus shorti" from Venezuela and concluded that the identification was based on a misinterpretation of a buccal appendix and two thoracic sclerites. We rename it Llanoterus shorti, a new genus and a new combination, based on gonocoxas, lateroterguites, gonocoxoesternites, pronotum, prosternal process and metaventrites. We illustrate habitus, female and male genitals, and other thoracic sclerites.
\end{abstract}

Key words: aquatic coleopteran, new combination, new genus, Noterini, Suphisellus
RESUMEN: Suphisellus es un género neártico de coleópteros acuáticos que ha sido identificado erróneamente en los trópicos. Analizamos "Suphisellus shorti" de Venezuela y concluimos que la identificación se basó en una interpretación errónea de un apéndice bucal y dos escleritos toráxicos. Lo llamamos Llanoterus shorti, un nuevo género y una nueva combinación, basada en la gonocoxa, lateroterguito, gonocoxoesternito, pronoto, apófisis prosternal y metaventrito. llustramos el habitus, genitales femeninos y masculinos, y otros escleritos toráxicos.

Palabras clave: Coleoptera acuática, nueva combinación, nuevo género, Noterini, Suphisellus.
El género Suphisellus es un grupo neártico de coleópteros acuáticos con especies mal identificadas en el neotrópico. Fue sugerido por Crotch (1873) en su revisión de las especies de Dytiscidae de los Estados Unidos, separando dos especies colocadas en el género Suphis Aube, 1836, como S. bicolor y S. lineatus. Aun cuando describió la especie Suphisellus punticollis, Crotch nunca registró formalmente el género. Young (1979), describió formalmente el género Suphisellus, y lo registró como Suphisellus Crotch, 1873.

Muchas especies determinadas como Canthydrus por Sharp (1882), se encontraron mezcladas con las especies de Hydrocanthus (Sharp, 1882) y fueron separadas por Crotch (Sharp, 1882), debido a la gran similitud de algunos caracteres, entre ellos, la apófisis prosternal, las sugirió como Suphisellus. Por ello, muchas especies descritas como Canthydrus en su mayoría, Noterus e Hydrocanthus, fueron determinadas posteriormente como Suphisellus en el neotrópico. Actualmente existe un número de especies de Noteridae que han sido determinadas como
Suphisellus, con base a un apéndice bucal (palpo maxilar) y dos escleritos toráxicos (pronoto y apófisis prosternal).

Suphisellus shorti García, Benetti y Camacho, 2012, se describió con base a algunos caracteres como el ápice del cuarto palpómero maxilar y la línea submarginal del margen lateral del pronoto. La línea submarginal pronotal, es un carácter plesiomorfico, que no define, ya que es un carácter compartido por otros géneros de Noteridae. En el género Suphisellus, este carácter ha sido tomado como un carácter genérico, lo cual, no es así. Este género necesita una revisión urgente, debido a que concentra especies que pertenecen a otras taxa diferentes de Suphisellus. Esta línea no es de longitud constante, y muchas veces se continúa de puntos hasta el margen anterior del pronoto. En S. shorti, esta línea es casi imperceptible y se extiende solo cerca del ángulo posterolateral y no es constante ya que no aparece en la mayoría de los ejemplares estudiados. Lo que determinó que es un carácter apomórfico o derivado, que está tendiendo a desaparecer, y son los caracteres apomórficos los que 
determinan las nuevas definiciones taxonómicas. Miller (2009), en su filogenia de la familia Noteridae, resaltó una serie de caracteres que identificaron al género Suphisellus, dichos caracteres no fueron tomados en cuenta en 2012, al momento de describir la especie S. shorti. En dicha filogenia se resaltaron los caracteres de la hembra, como la gonocoxa, lateroterguito y gonocoxoesternito. Estos caracteres no fueron utilizados por Young (1979) en la revisión de las especies de Suphisellus del norte de Colombia. Estos caracteres difirieron notablemente, cuando fueron comparados con la especie S. shorti. La característica morfológica externas y la morfología de los escleritos genitales, de la hembra, mostraron una serie de incompatibilidades, que sugirieron una pronta revisión para la especie S. shorti. Además, el estudio que se realizó sobre los escleritos ventrales mostró claramente la morfología de otro género diferente a Suphisellus.

Esta investigación tuvo por objetivo la separación, creación y descripción de un nuevo género, diferente de Suphisellus y una nueva combinación en la especie $S$. shorti, a partir de la revisión de la especie tipo.

\section{MATERIALES Y MÉTODOS}

Material estudiado: Se examinaron 331 ejemplares incluyendo holotipo y paratipos de S. shorti. Todo el material examinado se encuentra depositado en las colecciones del Museo de Artrópodos de La Universidad del Zulia, Maracaibo, Estado Zulia, Venezuela y del Museo del Instituto de Zoología Agrícola de la Universidad Central de Venezuela.

Preparación de genitalia: Se tomaron algunos ejemplares macho y hembra de la especie $S$. shorti, utilizando paratipos y material extra. Los ejemplares fueron previamente colocados en agua tibia por tres a cuatro minutos, lo que permitió ablandar los escleritos genitales. La extracción se realizó utilizando dos aplicadores con punta minuta, para evitar el daño de los ejemplares. Una vez extraídos, fueron colocados en solución de $\mathrm{KOH}$ al 10\%, para aclararlos y eliminar los restos de tejido adiposo conjuntivo adheridos a la estructura esclerotizada.

En la extracción de genitalia se utilizó un micro estereoscopio 100x de resolución, marca Leica M10. La estructura genital es conservada en solución alcohólica de glicerina, para posteriores observaciones y otras fueron montadas en portaobjetos, utilizando como medio de montaje Euparal. En el tratamiento sistemático, la identificación de estructura como los escleritos genitales y toráxicos se utilizó el mismo micro estereoscopio, pero con oculares 20x, aumentando la resolución a 120x. Para la fotografía se utilizo una cámara Nikon. Las partes fueron dibujadas, utilizando una cámara lúcida adaptada al micro estereoscopio. Las imágenes fueron mejoradas utilizando un programa de dibujo vectorial, Inkscape para Windows versión 0.91-1.

\section{RESULTADOS}

\section{Tratamiento taxonómico:}

Familia Noteridae Thomson 1860

Subfamilia Noterinae 1860

Tribu Noterini 1860

Género Llanoterus García y Camacho, gen. nov.

Especie tipo: Suphisellus shorti (García, Benetti y Camacho 2012)

Diagnosis: Forma ovo redondeada y robusta, dorsalmente muy convexa (Fig. 1A), con los márgenes laterales continuo y el ápice elitral redondeado. El ángulo posterolateral del pronoto presenta una línea submarginal por encima del margen pronotal, casi imperceptible (Fig. 2).

Descripción: Cabeza ancha y micro reticulada con celdas irregulares en el integumento. Clípeo con una pseudofranja transversal. Palpos maxilares con el último palpómero más ancho y más largo, que los que precede, con una hendidura muy profunda en el ápice, separando dos pseudo triángulos de longitudes desiguales. Pronoto tan ancho como la mitad de su longitud (Fig. 1A). Margen lateral con una muy fina y muy corta línea submarginal que nace del ángulo posterolateral pronotal y se extiende hasta muy cerca de la base, solo visible en algunos ejemplares (Fig. 2). Borde marginal del margen lateral pronotal micro aserrado. Élitro con el ápice elitral redondeado (Fig. 1A). Prosterno suavemente sinuoso en el margen anterior (Fig. 3). Disco prosternal, con una corta hilera de setas centrales, y una serie de setas o pequeñas espinas cortas distribuidas sobre la superficie, extendiéndose dos tercios de la longitud de la apófisis prosternal (Fig. 1B). Apófisis prosternal (Fig. 3) corto y ancho, de márgenes rectos, pseudorectangular con una constricción basal de la apófisis (Fig. 1B, 3). Margen apical suavemente redondeado en el macho (Fig. 3) y suavemente depreso en la hembra (Fig. 3). Superficie de la apófisis levemente depreso longitudinalmente (Fig. 1B y 
3). Metasterno angosto en el ápice, levemente depreso longitumedialmente, formando dos lóbulos metasternales distales lateralmente (Fig. 3). Placa coxal ancha de lados casi paralelos (Fig. 3). Lóbulos postcoxales anchos y truncados apicalmente, con una serie de espinas o setas gruesas en el ápice (Fig. 3). Abdomen con cinco esternito abdominales visibles (Fig. 1B). Ápice del séptimo esternito redondeado, formando un vértice longitudinal. Octavo esternito oculto por el séptimo, impreso longitudinalmente. Patas con el fémur anterior y medio no ensanchado. Margen apical de la tibia anterior con una espina robusta, larga, fuertemente curvada en el ápice. Tarso anterior con el primer tarsómero largo, ancho y triangular. Protarso adjunto en la base de la espuela protibial. Trocánter posterior ancho y largo, cerca de un tercio de la longitud del fémur posterior. Fémur posterior con la base angosta, peciolada cerca de un tercio de su longitud. Espuela metatibial anterior no serrada. Genitalia del macho (Fig. 4) con el edeago fuertemente arqueado, ápice levemente aguzado, parámero izquierdo cóncavo de igual longitud que el edeago, con una hilera de largos pelos sobre dos tercios del margen ventral, parámero derecho corto y ensanchado con la base angosta. Genitalia de la hembra (Fig. 5), gonocoxa con la cara externa convexa y la cara interna cóncava, de ápice extendido y agudo. Lateroterguitos curvados en la base y suavemente sinuoso distalmente, formando dos lóbulos pequeños. Base de lateroterguitos extendiéndose más allá de la base gonocoxal. Gonocoxoesternito con dos apodemas, uno ventral corto y uno dorsal largo ligeramente arqueado.

Etimología: se coloca el nombre dedicado a los Llanos de Venezuela, lugar de colecta de los ejemplares.

Especie tipo: Llanoterus shorti (García, Benetti y Camacho, 2012) comb. nov. Género masculino.

Material tipo: Holotipo $\widehat{\partial}$, Venezuela, Estado Apure, Municipio Biruaca, Fundo La Guama, $20 \mathrm{~km}$ al noroeste de San Fernando, M. García col., 20.viii.2006 (7042'16.57" N y 67032'27.44" W) elevación 51m (MALUZ 060069). Paratipos: 45 exs. $\widehat{\partial} \widehat{\partial}$, misma localidad de holotipo, 10.viii.1998, (MALUZ 06070-06115). 75 exs. 9 , , misma localidad de holotipo, 10.viii.1998, (MALUZ 06116-06191). 5 exs. $\widehat{\partial} \widehat{\partial}$, misma localidad de holotipo, 10.viii.1998, depositados en el Museo del Instituto de Zoología Agrícola de la Universidad Central de Venezuela, (MALUZ 06192-06296). 25 exs. 우오, misma localidad del holotipo, 10.viii.98, depositados en el Museo del Instituto de

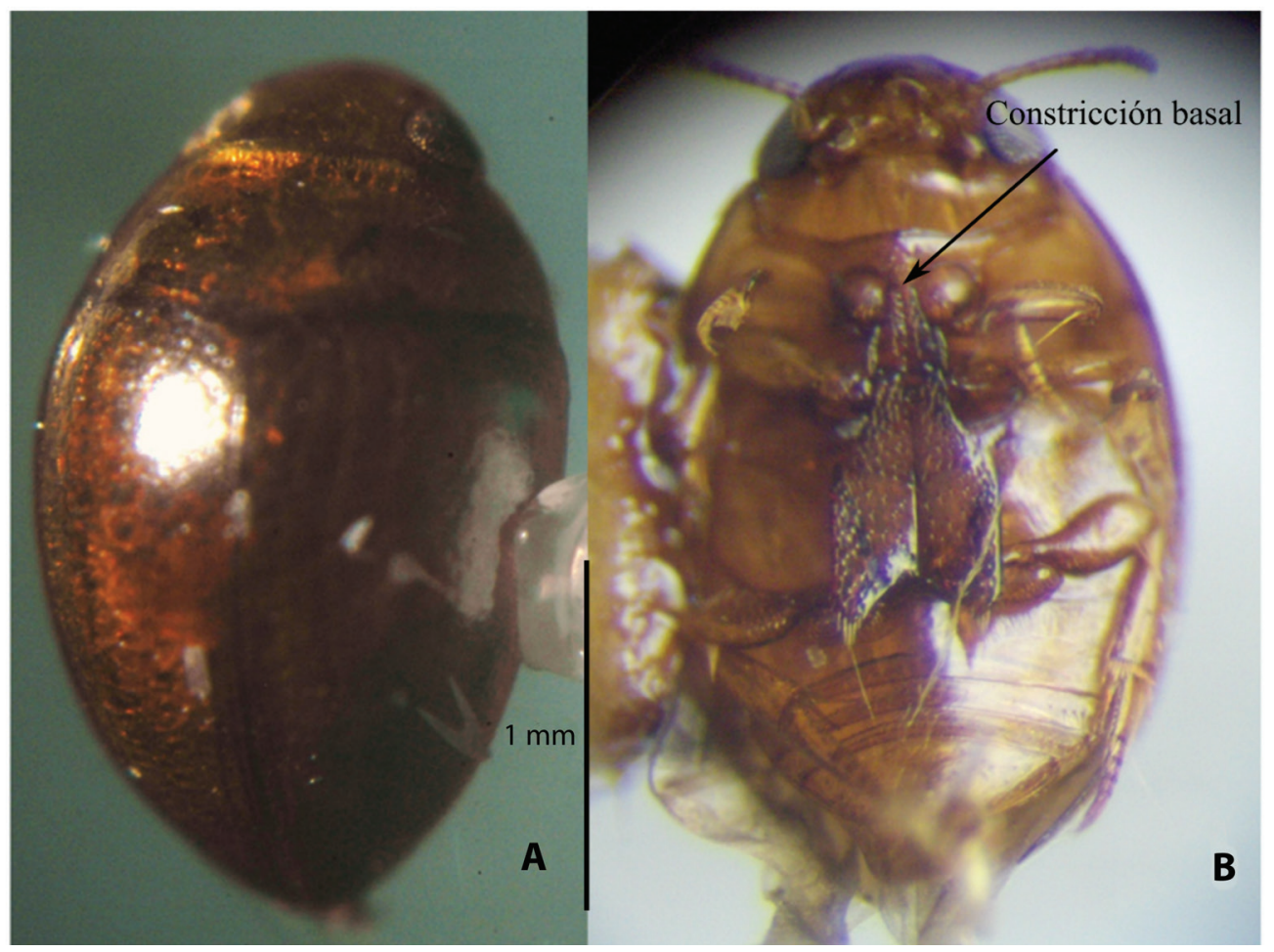

Fig. 1. L. shorti: A) Habitus vista dorsal y B) vista ventral. 


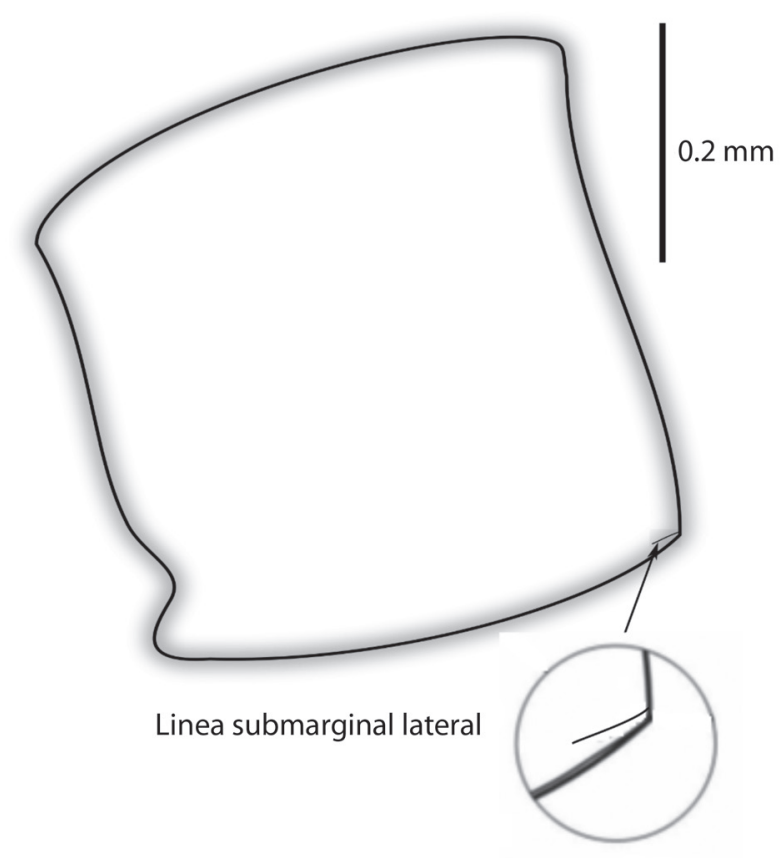

Fig. 2. Margen lateral del Pronoto de L. shorti.

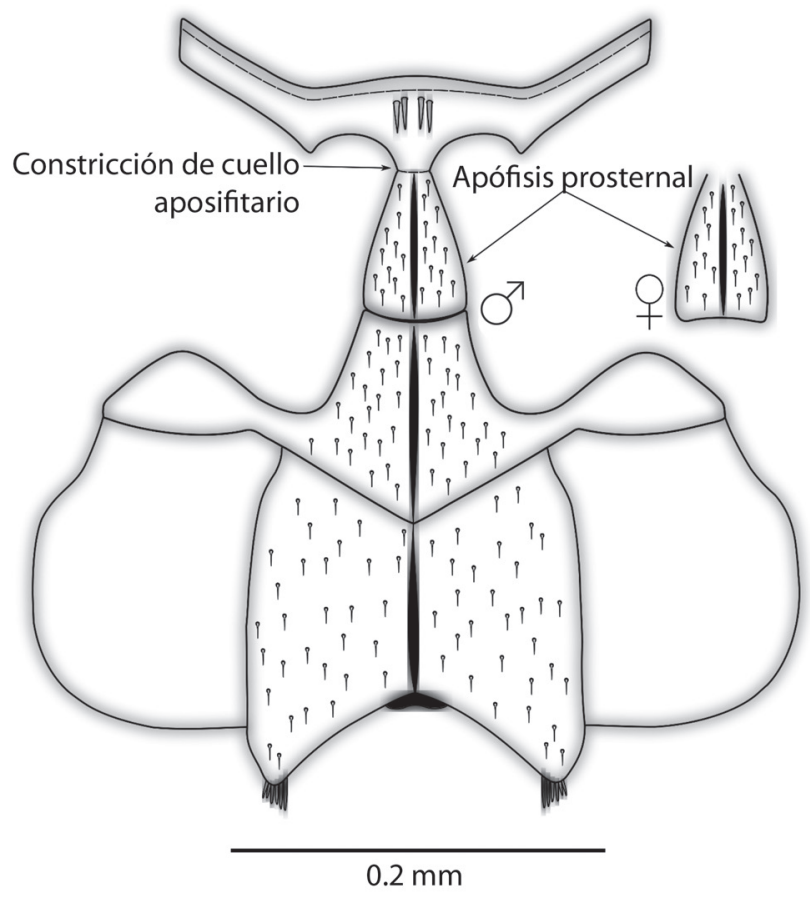

Fig. 3. Escleritos ventrales de $L$. shorti gen. nov.

Zoología Agrícola de la Universidad Central de Venezuela, (MALUZ 06197-06220). 80 exs., mismo dato del holotipo (MALUZ 06221-06300); 70 exs. (MALUZ) misma localidad del holotipo, 22.v.2007, (MALUZ 06301-06370); 50 exs.

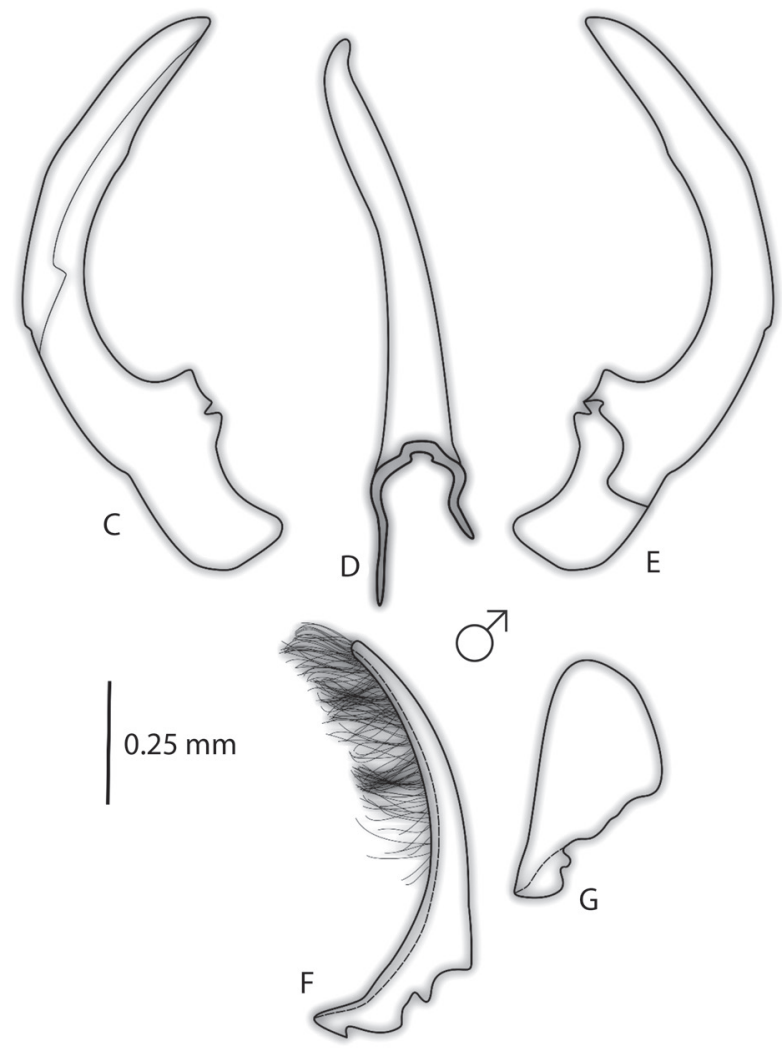

Fig. 4. Genitalia $\widehat{\partial}$ de $L$. shorti gen. nov.: C) Edeago vista lateral derecho, D) vista ventral, E) lateral izquierdo, F) parámero izquierdo vista lateral y G) parámero derecho vista lateral.

Venezuela, Guárico estado Miranda Municipio Puerto Miranda, M. García col., 24.xii.2004, (7054'31.06" N y 67028'22.49" W) elevación 50m, (MALUZ 06371-06420). Todos los tipos, nuevo etiquetado amarillo, Comb. Nov.

\section{DISCUSIÓN}

Llanoterus gen. nov., ha sido colocado dentro de la tribu Noterini, con caracteres sistemáticos compartidos por otros géneros de la subfamilia Noterinae, lo cual en el pasado llevo a confundirla con el género Suphisellus.

En Llanoterus gen. nov., se observaron caracteres, como la hendidura en él ápice del cuarto palpómero maxilar, pero este carácter bífido presente en las especies de Suphisellus, también es compartida con las especies de Suphis y en menor grado entre las especies de Mesonoterus y Liocanthydrus.

La línea submarginal lateral, que no solamente es inconclusa, sino muy reducida, casi imperceptible y solo aparece en tres de cada diez ejemplares examinados. 


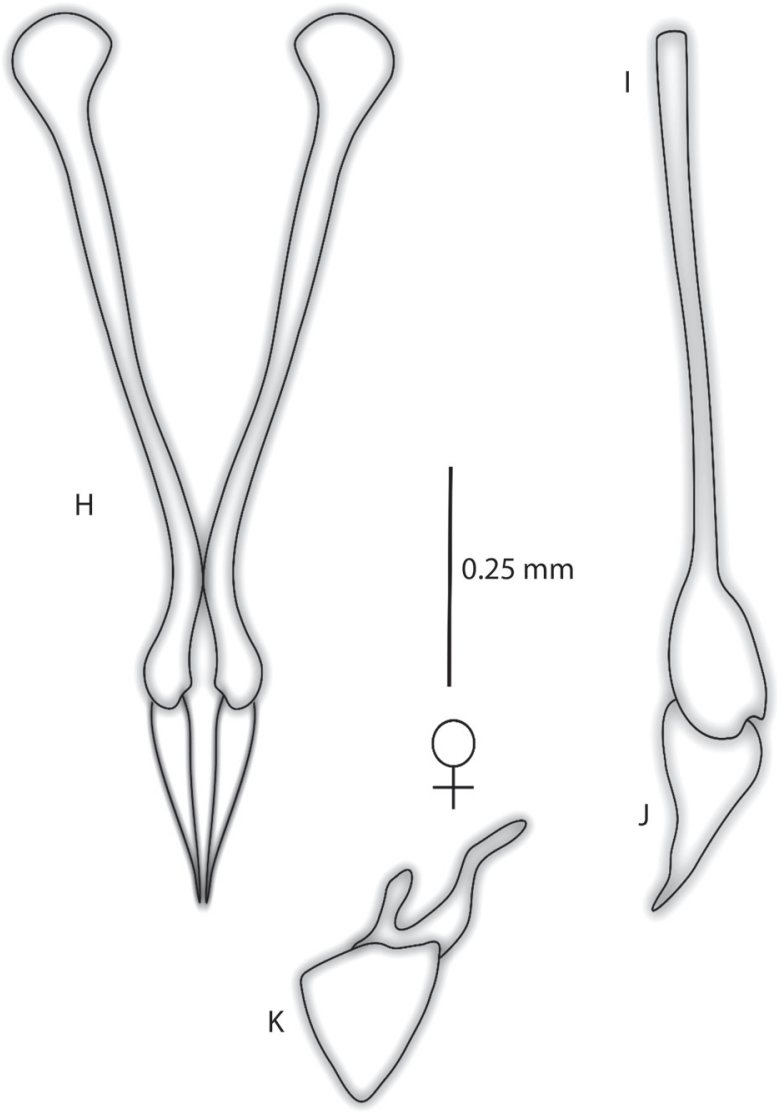

Fig. 5. Genitalia $q$ de L. shorti gen. nov.: H) vista dorsal, I) lateroterguito y J) gonocoxa vista lateral izquierda, K) gonocoxoesternito.

Hemos observados claramente, que es un carácter derivado de la línea submarginal, que en realidad es parte del margen lateral y su longitud varía según el género en la familia Noteridae. Este carácter reducido de la línea submarginal se tomó como determinante para el nuevo género Llanoterus. En Llanoterus gen. nov., esta línea es débil, casi imperceptible, nace en la base del ángulo posterolateral del pronoto, pero solo se extiende menos de $1 / 10$, de la longitud del pronoto, para luego desaparecer (Fig. 2). En algunas especies de Noteridae se presenta de forma muy variada esta línea submarginal. En los géneros Pronoterus, Mesonoterus y Prionohydrus, esta línea submarginal se extiende longitudinalmente de $8 / 10$ a 9/10 la longitud del pronoto, y de forma inconclusa toca el margen del ángulo anterolateral del pronoto. En Pronoterus se encuentra acompañado de puntos laterales sobre una débil depresión en su recorrido. En Mesonoterus no se presentan puntuaciones laterales, pero si sobre la línea, al igual que en Prionohydrus.
En los géneros Hydrocanthus, Liocanthydrus y Canthydrus, esta línea submarginal, se extiende completamente hasta llegar a tocar el margen anterolateral del pronoto, de forma variada, lo que lo lleva a formar parte del margen lateral. En Hydrocanthus se extiende en forma ascendente, en Liocanthydrus se extiende paralelamente al borde marginal, con una sinuosidad preapical, antes de tocar el margen anterior y en Canthydrus se extiende de forma paralela al borde marginal, hasta tocar el margen anterior. Esta línea submarginal representa el borde superior lateral del margen pronotal. Pero en el género Suphisellus, esta línea se presenta, en forma ascendente e inconclusa, que solo llega hasta la mitad de la longitud pronotal. Algunos autores manejan este carácter como un pliegue lateral (Young 1979). Este estudio con especies de otros géneros de Noteridae, permitió constatar, que la línea submarginal, es un carácter plesiomorfico, y se encuentran muy compartidos en la familia.

Llanoterus gen. nov., comparte otro carácter principal, la apófisis prosternal ancha, con otras especies de eunoterinae de los géneros Suphis y Suphisellus, por su forma abiertamente bífida (en algunas especies solo levemente hendida). La apófisis prosternal (Fig. 1B, 3), es un esclerito ventral, mejor conocido como proceso prosternal, y representa otro carácter de discusión sistemática. Este esclerito ventral, presenta formas muy variadas, en la familia Noteridae: forma ancha, larga, corta, con márgenes laterales rectos o sinuosos y margen apical, recto, sinuoso o redondeado. En Llanoterus gen. nov., este esclerito es corto y el ápice es menos de dos veces el espacio intercoxal (Fig. 1B, 3). La apófisis prosternal presenta márgenes laterales rectos con una pequeña constricción en la base (Fig. 1B, 3). El margen apical es suavemente redondeado en machos y algo depreso en la hembra. La superficie de la apófisis es débilmente depreso longitumedialmente (Fig. 1B, 3). En Hydrocanthus, Liocanthydrus, Tonerus y Canthysellus, se presenta de forma ancha y de márgenes variados, pero de rasgos característicos casi similares entre sí. En Suphis y Noterus, fueron igualmente anchos, pero de formas más redondeadas en los márgenes. En Pronoterus, Mesonoterus y Prionohydrus, de rasgos angostos y cortos, de márgenes sinuosos y ápice redondeados o acuminado. En Llanoterus gen. nov., Canthydrus y Suphisellus la forma es pseudotriangular, anchos y largos, exceptuando a Llanoterus gen. nov., que es algo más corto y en los otros dos géneros se presenta sin constricción basal, de márgenes rectos y ápice variable.

La estructura genital de la hembra en Llanoterus shor$t i$, la gonocoxa (Fig. 5H, 5J) presenta el ápice alargado y agudo y el lateroterguito menos largo y más curvo que en Suphisellus. Los dos apodemas en el gonocoxoesternito (Fig. 5K) en Llanoterus gen. nov., presentan posiciones 
diferentes, con respecto a Suphisellus. El apodema corto se encuentra en la posición lateral y el largo en la posición central, en Suphisellus, ocurre lo contrario, el apodema corto se encuentra en la posición central y el largo en la posición lateral (Miller 2009). Se ha observado, que desde el punto de vista de genitalia de la hembra, existe más relación con el género Canthydrus, que con Suphisellus, (Miller 2009).

Los escleritos genitales del macho de Llanoterus gen. nov., presentan cierta similitud con respecto a la forma arqueada del edeago con algunas especies de Suphisellus que erróneamente ha sido mal interpretada. Pero ayudado de genitalia de la hembra se hizo consistente y claramente definible. Los escleritos genitales de la hembra son tan determinantes y tan importantes como genitalia del macho, ya que ambos representan a la especie. Con esta investigación se espera una mayor utilidad de los rasgos característicos de genitalia de la hembra, como un factor de identificación, en la separación de taxa.

\section{AGRADECIMIENTOS}

A William Shepard de la Universidad de Berkeley, por su valiosa colaboración al hacerme llegar los artículos de interés relacionados con el manuscrito.

\section{REFERENCIAS}

Crotch, G. R. (1873). Revision of the Dytiscidae of the United States. Transactions of the American Entomological Society, 4, 383-424.

García, M., Benetti, C. J., \& Camacho, J. (2012). A new species of Suphisellus Crotch, 1873 (Coleoptera: Noteridae) from "Los Llanos", Venezuela. Zootaxa, 3298, 62-68.

Miller, K. B. (2009). On the Systematics of Noteridae (Coleoptera: Adephaga: Hydradephaga): Phylogeny, description of a new tribe, genus and species, and survey of female genital morphology. Systematics and Biodiversity, 7, 191-214.

Sharp, D. (1882). On aquatic carnivorous Coleoptera or Dytiscidae. The Scientific Transactions of the Royal Dublin Society, 2(1880-1882), 179-1003.

Young, F. N. (1979). Water beetles of genus Suphisellus Crotch in the Americas north of Colombia (Coleoptera: Noteridae). The Southwestern Naturalist, 24(3), 409-429. 